\title{
The Quantum Signature of Chaos through the Dynamics of Entanglement in Classically Regular and Chaotic Systems
}

\author{
L.Y. CHEW ${ }^{a, *}$ AND N.N. CHUNG ${ }^{b}$ \\ ${ }^{a}$ Division of Physics and Applied Physics, School of Physical and Mathematical Sciences \\ Nanyang Technological University, 21 Nanyang Link, Singapore 637371 \\ ${ }^{b}$ Temasek Laboratories, National University of Singapore, Singapore 117508
}

\begin{abstract}
We demonstrate the dependence of the entanglement dynamics on the global classical regime of regular, mixed and chaotic dynamics with initial squeezed coherent states. By squeezing the initial states, we are able to enhance or suppress the entanglement entropy of the dynamically generated quantum states relative to those produced via initial coherent states. This suggests the possibility of exploiting a judicious selection of initial squeezed states to create quantum states with enhanced entanglement entropy dynamically for the purpose of robust quantum information processing.
\end{abstract}

PACS: 05.45.Mt, 03.67.Bg, 03.67.Mn

\section{Introduction}

Quantum chaos is a useful paradigm for the investigation of the quantum mechanics of nonlinear dynamical systems. Through the notion of quantum-classical correspondence, quantum chaos investigates how the appearance of chaos in the classical domain can affect the corresponding world of the quantum. For example, classical chaos is known to leave its imprints in quantum properties such as the energy level statistics, the scarring, or the localization of the wave function [1]. With recent interest in the study of entanglement between bipartite quantum systems, one naturally ask how chaos can influence the dynamics of the entanglement entropy within these quantum systems. At the present moment, there is active research into the answers to this question [2-7].

While the effect of entanglement has been described as "spooky", it basically indicates the presence of quantum correlations between the subsystems of a composite quantum system. From the perspective of quantum complexity, entanglement plays an important role in creating the whole of a quantum system which is more than the sum of its parts. In terms of practical applications, its non-classical feature has enabled unconventional information processing capabilities such as superdense coding, quantum teleportation and quantum cryptography [8], which are beyond the classical approach. Entanglement thus serves as an important resource in quantum information processing. To exploit it, it is necessary to first generate quantum states that are entangled. An approach to create entangled quantum states is through the dynamics of coupled quantum anharmonic oscillator systems [9-11],

\footnotetext{
* corresponding author; e-mail: lockyue@ntu.edu.sg
}

which can be implemented experimentally by means of optical fibres with the Kerr nonlinearities [12]. This approach of generating entangled quantum states is of interest in this paper.

Recently, there are diverse studies on the correspondence between dynamical chaos and entanglement in systems such as the coupled kicked tops [2, 7], the Rydberg molecules [5] and interacting spin systems [6]. These studies have found that when the classical system is more chaotic, the corresponding quantized system is more entangled and possesses a higher entanglement production rate, albeit there are some notable exceptions [13, 14]. In addition, the frequency of oscillation of the entanglement dynamics increases as the classical system becomes more chaotic. More significantly, these researches have found that the behavior of the entanglement dynamics is dependent on the local dynamical structure of the corresponding classical phase space $[2,4,5]$. While this may indicate that a universal quantum-classical correspondence in terms of entanglement dynamics is impossible, our research has nonetheless uncovered that it is possible for the entanglement dynamics to depend solely on the global classical dynamical regime [3]. It is important to note that all the above research have been performed with coherent state as initial state, since coherent state is closest to the classical state. These researches have not, however, considered the case of squeezed coherent state.

In this paper, we have further explored into the dependence of entanglement dynamics on the global classical dynamical regime by squeezing the initial separable coherent states. As before, this study is performed on a coupled quartic oscillator system which is able to exhibit regular, mixed and purely chaotic dynamics. By quantizing this oscillator system, we shall relate the entanglement dynamics derived from the initial squeezed states to 
the corresponding dynamics in the classical phase space. Our goal is to examine the effect of squeezing the initial states on: (i) the behavior of the entanglement dynamics, and (ii) the dependence of the entanglement dynamics on the global classical dynamical regime.

\section{Coupled quartic oscillator system with initial squeezed coherent states}

The Hamiltonian of the coupled quartic oscillator system of interest in this paper has the following form [15]:

$$
H=\frac{1}{2}\left(p_{1}^{2}+p_{2}^{2}\right)+3 x_{1}^{4}+x_{2}^{4}-\lambda x_{1}^{2} x_{2}^{2},
$$

where $p_{1}$ and $p_{2}$ are the momenta, $x_{1}$ and $x_{2}$ are the position, and $\lambda$ is the coupling constant of the oscillator. Previously, we have shown that depending on the value of $\lambda$, the classical dynamics of this coupled anharmonic oscillator system can exhibit: (a) purely regular dynamics, (b) a mixture of regular and chaotic dynamics, and (c) purely chaotic dynamics [3].

In order to evaluate the entanglement dynamics, we first quantize Eq. (1) by replacing $x_{k}$ and $p_{k}$ with their corresponding operators $\hat{x_{k}}$ and $\hat{p_{k}}$. Let us note that from now on, the subscript $k \in\{1,2\}$. The time evolved state $|\psi(t)\rangle$ of the resulting two-coupled quantum quartic oscillator system is then determined by computing the following Schrödinger equation:

$$
\mathrm{i} \hbar \frac{\mathrm{d}}{\mathrm{d} t}|\psi(t)\rangle=\sum_{n_{1}=0}^{M} \sum_{n_{2}=0}^{M} \hat{H}\left|n_{1}, n_{2}\right\rangle\left\langle n_{1}, n_{2} \mid \psi(t)\right\rangle,
$$

numerically. Let us note that we have expressed Eq. (2) in terms of the number basis $\left|n_{1}, n_{2}\right\rangle$ of the harmonic oscillator. In other words, $\left|n_{k}\right\rangle$ is the eigenstate of $\hat{H}_{k}={\hat{p_{k}}}^{2} / 2+{\hat{x_{k}}}^{2} / 2$. The basis size of the computation is limited to $M$.

In our previous work [3], the initial states are product of coherent states $\left|\alpha_{1}\right\rangle \otimes\left|\alpha_{2}\right\rangle$. In this paper, we investigate the effects of squeezing these initial states. Squeezed states are quantum states wherein the quantum noise in one of its field quadratures has been significantly suppressed. Currently, squeezed states are routinely produced in laboratories using both solid state and semiconductor lasers, as well as within high- $Q$ cavities. A squeezed coherent state is in fact an equivalence class of minimum uncertainty packets to the coherent state [16]. By squeezing each mode of our initial coherent states $|\psi(0)\rangle$ separately, our initial squeezed coherent states can be expressed as $\left|\alpha_{1}, \xi_{1}\right\rangle \otimes\left|\alpha_{2}, \xi_{2}\right\rangle$. The single-mode squeezed coherent state $\left|\alpha_{k}, \xi_{k}\right\rangle$ is defined as

$$
\left|\alpha_{k}, \xi_{k}\right\rangle=\hat{D}\left(\alpha_{k}\right) \hat{S}\left(\xi_{k}\right)|0\rangle,
$$

where

$$
\hat{D}\left(\alpha_{k}\right)=\exp \left(\alpha_{k} \hat{a}_{k}^{\dagger}-\alpha_{k}^{*} \hat{a}_{k}\right)
$$

and

$$
\hat{S}\left(\xi_{k}\right)=\exp \left(\xi_{k}^{*} \hat{a}_{k} \hat{a}_{k}-\xi_{k} \hat{a}_{k}^{\dagger} \hat{a}_{k}^{\dagger}\right) .
$$

First, the variable $\alpha_{k}$ in the displacement operator $\hat{D}$ gives the connection between the quantum and the classical domain. More specifically, the center of the squeezed coherent state corresponds precisely to the point $\left(x_{1}, p_{1}, x_{2}, p_{2}\right)$ in the classical phase space, with $\alpha_{k}=\left(x_{k}+\mathrm{i} p_{k}\right) / \sqrt{2}$. Second, the variable $\xi_{k}=$ $r_{k} \exp \left(\mathrm{i} \theta_{k}\right)$ in the squeezing operator $\hat{S}$ gives the magnitude $r_{k}$ and the angular direction $\theta_{k}$ of squeezing the field quadratures of the quantum state. Let us note that $\hat{a}_{k}$ and $\hat{a}_{k}^{\dagger}$ are the usual annihilation and creation operator. The squeezed coherent states $\left|\alpha_{k}, \xi_{k}\right\rangle$ can be expanded in terms of the number basis as follows [16-18]:

$$
\left|\alpha_{k}, \xi_{k}\right\rangle=\sum_{n_{k}=0}^{\infty} c_{k}\left(n_{k}\right)\left|n_{k}\right\rangle,
$$

where the coefficients

$$
\begin{aligned}
& c_{k}\left(n_{k}\right)=N_{k}\left[n_{k} ! \cosh \left(r_{k}\right)\right]^{-1 / 2}\left[\frac{1}{2} \mathrm{e}^{\mathrm{i} \theta_{k}} \tanh \left(r_{k}\right)\right]^{n_{k} / 2} \\
& \times \mathcal{H}_{n}\left(\gamma_{k}\left[\mathrm{e}^{\mathrm{i} \theta_{k}} \sinh \left(2 r_{k}\right)\right]^{-1 / 2}\right) .
\end{aligned}
$$

Let us note that $\mathcal{H}_{n}$ denotes the Hermite polynomial,

$$
\gamma_{k}=\alpha_{k} \cosh \left(r_{k}\right)+\alpha_{k}^{*} \mathrm{e}^{\mathrm{i} \theta_{k}} \sinh \left(r_{k}\right)
$$

and

$$
N_{k}=\exp \left(-\frac{1}{2}\left|\alpha_{k}\right|^{2}-\frac{1}{2}\left(\alpha_{k}^{*}\right)^{2} \mathrm{e}^{\mathrm{i} \theta_{k}} \tanh \left(r_{k}\right)\right) .
$$

With our initial squeezed coherent state expressed in terms of the number basis

$$
|\psi(0)\rangle=\sum_{m_{1}=0}^{\infty} \sum_{m_{2}=0}^{\infty} c_{1}\left(m_{1}\right) c_{2}\left(m_{2}\right)\left|m_{1}, m_{2}\right\rangle,
$$

we then integrate Eq. (2) to obtain the time-evolved state $|\psi(t)\rangle$, from which we form the density matrix $\hat{\rho}(t)$ of the two-coupled quantum quartic oscillator system

$$
\hat{\rho}(t)=|\psi(t)\rangle\langle\psi(t)| .
$$

Since our state is pure and protected from the decohering environment, the von Neumann entropy is an applicable measure of quantum entanglement. This has allowed us to employ the time variation of the von Neumann entropy

$$
S_{\mathrm{VN}}(t)=-\operatorname{Tr}\left(\hat{\rho}_{1}(t) \ln \hat{\rho}_{1}(t)\right)
$$

to determine the entanglement dynamics of our coupled oscillator system. Let us note that

$$
\hat{\rho}_{1}(t)=\operatorname{Tr}_{2}(\hat{\rho}(t))
$$

is the reduced density matrix of oscillator 1 . It is obtained by taking the partial trace over oscillator 2 on the density matrix $\hat{\rho}(t)$. Furthermore, replacing $\hat{\rho}_{1}(t)$ in Eq. (12) by the reduced density matrix of oscillator 2

$$
\hat{\rho}_{2}(t)=\operatorname{Tr}_{1}(\hat{\rho}(t))
$$

makes no difference to the result of the entanglement entropy. 


\section{Effects of initial squeezing on the entanglement entropy}

In this section, we proceed to explore into the correspondence between the quantum and the classical domain through the entanglement entropy when the classical system displays regular, mixed and chaotic dynamics. For our system concerned, these dynamics are found to occur at $\lambda=0.4,0.8$ and 2.7 respectively.

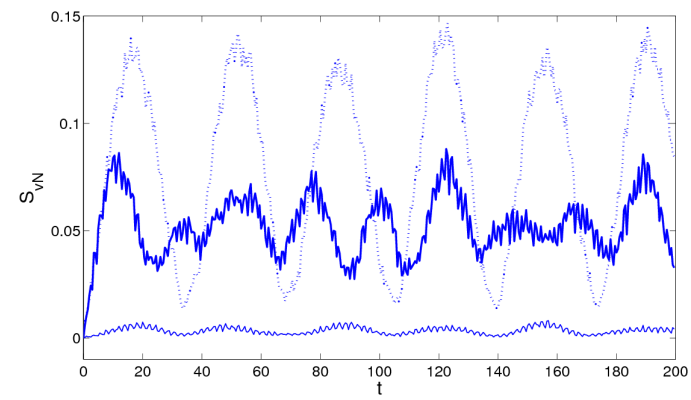

Fig. 1. Dynamics of the entanglement entropy for different initial squeezed coherent states with respect to the classically regular regime: $\xi_{1}=\xi_{2}=0$ (thick solid line); $\xi_{1}=\xi_{2}=0.4$ (solid line); and $\xi_{1}=-\xi_{2}=0.4$ (dotted line). Let us note that $\lambda=0.4$ and $M=115$.

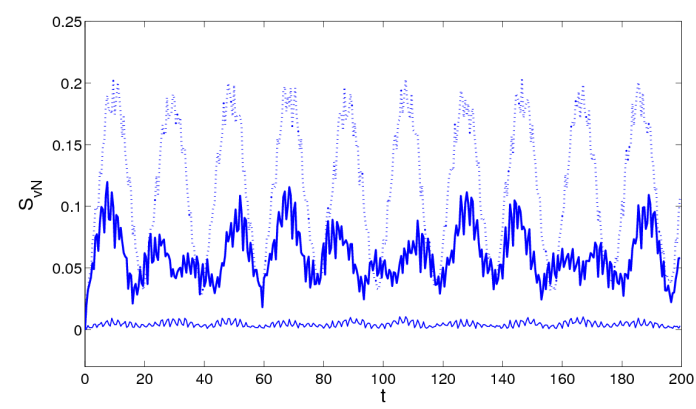

Fig. 2. Dynamics of the entanglement entropy for different initial squeezed coherent states with respect to the classically mixed regime: $\xi_{1}=\xi_{2}=0$ (thick solid line); $\xi_{1}=\xi_{2}=0.4$ (solid line); and $\xi_{1}=-\xi_{2}=0.4$ (dotted line). Let us note that $\lambda=0.8$ and $M=115$.

Our results are shown in Figs. 1, 2 and 3 for these three cases where we have considered the initial state to be the coherent state $\left(\xi_{1}=\xi_{2}=0\right)$, the symmetrically squeezed coherent state $\left(\xi_{1}=\xi_{2}=0.4\right)$ and the antisymmetrically squeezed coherent state $\left(\xi_{1}=-\xi_{2}=0.4\right)$. While we have found that squeezing the initial state symmetrically (or antisymmetrically) seems to reduce (or enhance) the entanglement entropy against that due to the initial coherent state, we observe the presence of an interesting pattern that is generic for the three cases. We uncovered that for each of the initial coherent or squeezed coherent states, the entanglement production is the highest in the pure chaos case, followed by the mixed case, and is lowest in the regular case. In addition, we also observe

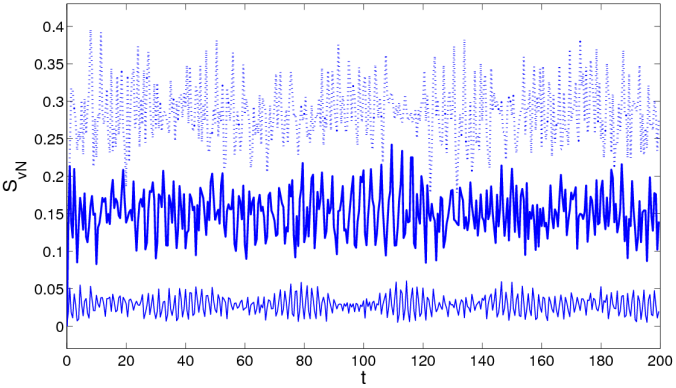

Fig. 3. Dynamics of the entanglement entropy for different initial squeezed coherent states with respect to the classically chaotic regime: $\xi_{1}=\xi_{2}=0$ (thick solid line); $\xi_{1}=\xi_{2}=0.4$ (solid line); and $\xi_{1}=-\xi_{2}=0.4$ (dotted line). Let us note that $\lambda=2.7$ and $M=115$.

that when the classical system becomes more chaotic, the frequency of oscillation of the entanglement dynamics increases. These results correspond to those reported in the literature. However, for each of the regular, mixed and chaotic case, we observe that almost identical results are obtained when different initial conditions are employed. This is a surprising result that differs from others in the literature. This result has led us to conclude in Ref. [3] that it is possible for the entanglement dynamics to depend solely on the global classical dynamical regime and not on the local classical behavior. Interestingly, our results here show that this conclusion remains true even when we subject our initial separable coherent states to the squeezing operation. Let us now illustrate the validity of our conclusion for initial squeezed coherent states by plotting the maximum entanglement entropy

$$
S_{\mathrm{M}}=\max _{t} S_{\mathrm{VN}}(t)
$$

and the mean entanglement entropy

$$
S_{\text {avg }}=\frac{1}{T} \int_{0}^{T} S_{\mathrm{VN}}(t) \mathrm{d} t
$$

against different initial states for the mixed case. To clearly demonstrate the dependence on global classical dynamical behavior, we have chosen the initial states to reside within the regular islands and the chaotic sea of the corresponding classical phase space [3]. As shown in Figs. 4 and 5, the $S_{\mathrm{M}}$ and $S_{\text {avg }}$ are constant (or nearly constant) for the respective initial states: the coherent states, the symmetrically squeezed coherent states or the antisymmetrically squeezed coherent states. These results have thus verified our claim.

While we have already noticed the outcome of squeezing the initial states on the entanglement dynamics (see Figs. 1 to 3 ) or on the quantity $S_{\mathrm{M}}$ and $S_{\text {avg }}$ (see Figs. 4 and 5), let us now give a more systematic examination on its effects on the entanglement entropy. In Fig. 6, we display our results of $S_{\mathrm{M}}$ and $S_{\text {avg }}$ against the squeezing paramter $\xi$, where we have assumed the initial squeezed coherent states to be real and symmetric, i.e. $\xi_{1}=\xi_{2}=\xi$ 


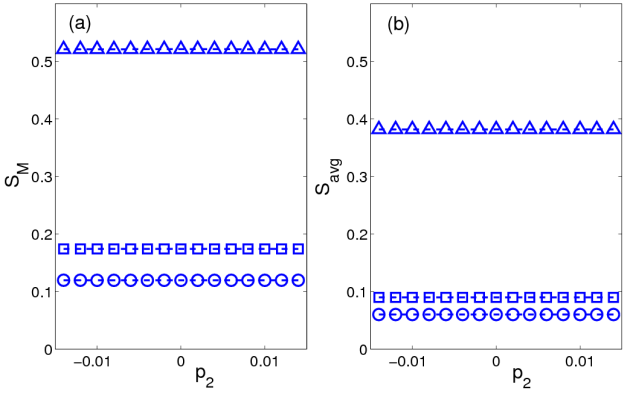

Fig. 4. Dependence of (a) $S_{\mathrm{M}}$ and (b) $S_{\text {avg }}$ on different initial state with $-0.015<p_{2}<0.015$. Let us note that the study here is performed with respect to the classically mixed regime. The parameters are: $\lambda=0.8$, $M=115, \xi_{1}=\xi_{2}=0$ (circles), $\xi_{1}=\xi_{2}=0.8$ (squares) and $\xi_{1}=-\xi_{2}=0.8$ (triangles).
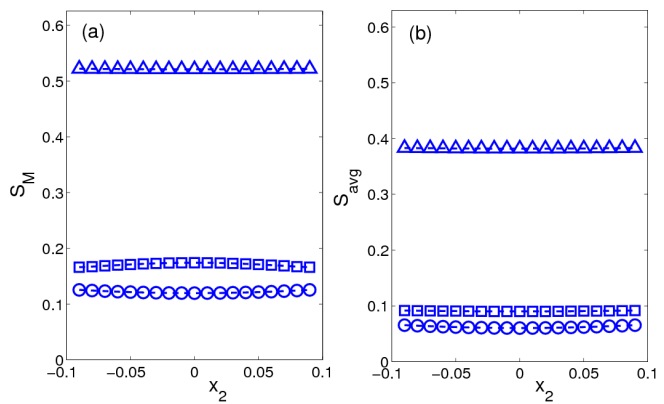

Fig. 5. Dependence of (a) $S_{\mathrm{M}}$ and (b) $S_{\text {avg on different }}$ initial state with $-0.1<x_{2}<0.1$. Let us note that the study here is performed with respect to the classically mixed regime. The parameters are: $\lambda=0.8, M=115$, $\xi_{1}=\xi_{2}=0$ (circles), $\xi_{1}=\xi_{2}=0.8$ (squares) and $\xi_{1}=-\xi_{2}=0.8$ (triangles).

and $\theta_{1}=\theta_{2}=0$ or $\pi$. We observe that $S_{\mathrm{M}}$ and $S_{\text {avg }}$ increase as $\xi$ becomes more negative. On the other hand, $S_{\mathrm{M}}$ and $S_{\text {avg }}$ first decrease and then increase as $\xi$ becomes more positive. This behavior of $S_{\mathrm{M}}$ and $S_{\text {avg }}$ occurs when the corresponding classical phase space is either regular, mixed or chaotic.

However, as indicated in Fig. 7, there is a reversal in behavior when the initial squeezed coherent states are real and antisymmetric. This arises when the corresponding classical phase space is regular and mixed. In these circumstances, the $S_{\mathrm{M}}$ and $S_{\mathrm{avg}}$ increase as $\xi$ becomes more positive; and first decrease and then increase as $\xi$ becomes more negative. On the contrary, the $S_{\mathrm{M}}$ and $S_{\text {avg }}$ follow the same pattern as that given in Fig. 6 for the fully chaotic case. Next, we investigate the influence of the angular orientation $\theta$ of the squeezing on $S_{\mathrm{M}}$ and $S_{\text {avg. }}$.

Figure 8 illustrates the situation of symmetric squeezing $\left(\theta_{1}=\theta_{2}=\theta\right)$ where we observe a monotonic increase of $S_{\mathrm{M}}$ and $S_{\text {avg }}$ against $\theta$. For the case of antisymmetric squeezing $\left(\theta_{1}=\theta_{2}-\pi=\theta\right)$ as illustrated in Fig. 9, we again find a reversal in the pattern of $S_{\mathrm{M}}$ and $S_{\text {avg }}$ for the classically regular and mixed regime relative to the chaotic regime. This result is similar to that shown in Fig. 7 above. Finally, we observe that in all cases, $S_{\mathrm{M}}$ (or $S_{\text {avg) }}$ is consistently the largest for the chaotic case, followed by the mixed case, and then the regular case.

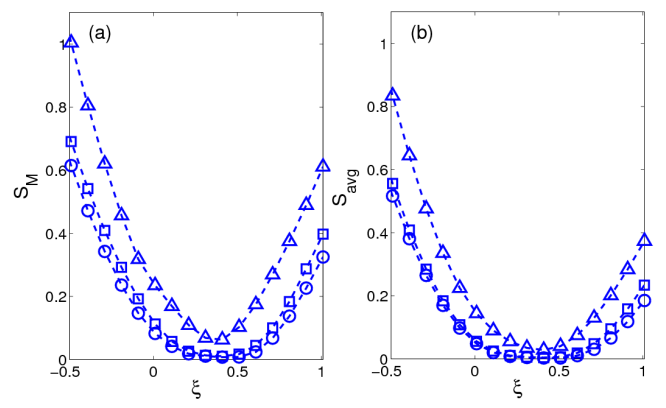

Fig. 6. Dependence of (a) $S_{\mathrm{M}}$ and (b) $S_{\text {avg }}$ on the squeezing parameter $\xi$ for symmetrically squeezed initial coherent state, i.e. $\xi_{1}=\xi_{2}=\xi$. We consider only cases where $\theta_{1}=\theta_{2}=\theta=0$ or $\pi$ so that $\xi$ is real. Let us note that $M=115, \lambda=0.4$ (circles), 0.8 (squares) and 2.7 (triangles).

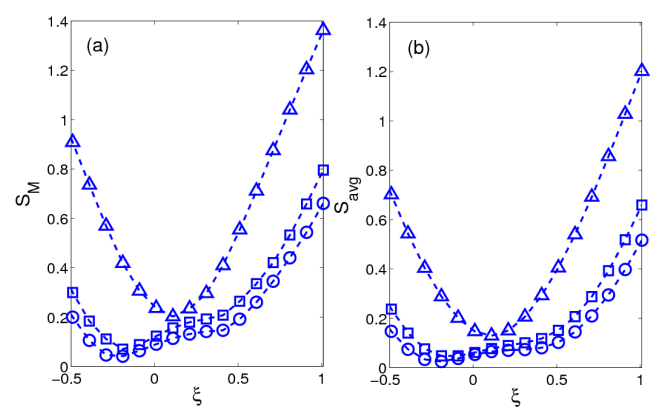

Fig. 7. Dependence of (a) $S_{\mathrm{M}}$ and (b) $S_{\text {avg }}$ on the squeezing parameter $\xi$ for antisymmetrically squeezed initial coherent state, i.e. $\xi_{1}=-\xi_{2}=\xi$. We consider only cases where $\theta_{1}=\theta_{2}=\theta=0$ or $\pi$ so that $\xi$ is real. Let us note that $M=115, \lambda=0.4$ (circles), 0.8 (squares) and 2.7 (triangles).

In summary, our results demonstrate that the dynamical production of entanglement entropy need not be enhanced through introducing quadrature squeezing in the initial coherent state. The entanglement can be suppressed under certain conditions. While the enhancement (or suppression) of $S_{\mathrm{M}}$ and $S_{\text {avg }}$ depends on the squeezing parameter $\xi$, our results show that these quantities are essentially independent of the displacement parameter $\alpha$, which signifies that the dependence of the entanglement dynamics on the global classical dynamical regime remains true even for the case of initial squeezed states.

\section{Conclusion}

In this paper, we have established the possibility of the entanglement dynamics to depend on the global classical 

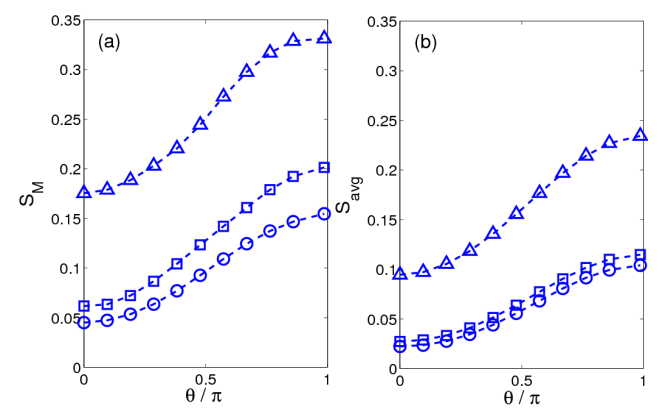

Fig. 8. Dependence of (a) $S_{\mathrm{M}}$ and (b) $S_{\text {avg }}$ on $\theta$ for symmetrically squeezed initial coherent state, i.e. $\theta_{1}=$ $\theta_{2}=\theta$. Let us note that $r_{1}=r_{2}=0.1, M=115$, $\lambda=0.4$ (circles), 0.8 (squares) and 2.7 (triangles).
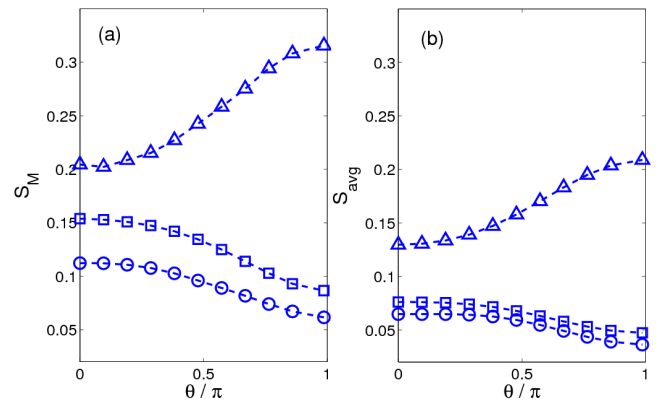

Fig. 9. Dependence of (a) $S_{\mathrm{M}}$ and (b) $S_{\text {avg }}$ on $\theta$ for antisymmetrically squeezed initial coherent state, i.e. $\theta_{1}=\theta_{2}-\pi=\theta$. Let us note that $r_{1}=r_{2}=0.1$, $M=115, \lambda=0.4$ (circles), 0.8 (squares) and 2.7 (triangles).

dynamical regime instead of the local classical behavior for initial squeezed coherent states. In particular, our results indicate that by squeezing the initial coherent states appropriately, an entangled state with enhanced entanglement entropy can be achieved. In fact, the entanglement entropy can be futher increased by exploiting quantum states which correspond to the classically chaotic regime. Thus, our results here have extended the advantage of "global" models [3], which in addition to being able to generate encoding subspaces that are stable against errors in the initial state preparation, are now able to produce quantum state with enhanced entanglement entropy through initial squeezing. We believe that these improved encoding subspaces will be important in the design of robust quantum information processing protocol in the near future.

\section{References}

[1] G. Casati, B. Chirikov, Quantum Chaos: between Order and Disorder, Cambridge University Press, 1995.

[2] M. Lombardi, A. Matzkin, Phys. Rev. E 83, 016207 (2011).

[3] N.N. Chung, L.Y. Chew, Phys. Rev. E 80, 016204 (2009).

[4] S. Zhang, Q. Jie, Phys. Rev. A 77, 012312 (2008).

[5] M. Lombardi, A. Matzkin, Europhys. Lett. 74, 771 (2006).

[6] M. Novaes, Ann. Phys. 318, 308 (2005).

[7] P.A. Miller, S. Sarkar, Phys. Rev. E 60, 1542 (1999).

[8] J. Audretsch, Entangled Systems: New Directions in Quantum Physics, Wiley-VCH, 2007.

[9] N.N. Chung, L.Y. Chew, Phys. Rev. A 80, 012103 (2009).

[10] N.N. Chung, L.Y. Chew, Phys. Rev. A 76, 032113 (2007).

[11] C.S. Hsue, J.L. Chern, Phys. Rev. D 29, 643 (1984).

[12] B. Yurke, D. Stoler, Phys. Rev. Lett. 57, 13 (1986).

[13] H. Fujisaki, T. Miyadera, A. Tanaka, Phys. Rev. E 67, 066201 (2003)

[14] M. Novaes, M.A.M. De Aguiar, Phys. Rev. E 70, 045201(R) (2004).

[15] M. Tomiya, N. Yoshinaga, S. Sakamoto, A. Hirai, Comput. Phys. Commun. 169, 313 (2005).

[16] D. Stoler, Phys. Rev. D 1, 3217 (1970).

[17] H.P. Yuen, Phys. Rev. D 13, 2226 (1976).

[18] J.J. Gong, P.K. Aravind, Am. J. Phys. 58, 1003 (1990). 